\title{
Large-Scale Activity Initiated BY Halo CMEs
}

\author{
I. Chertok ${ }^{1}$ and V. Grechnev ${ }^{2}$ \\ ${ }^{1}$ IZMIRAN, Troitsk, Russia, email: ichertok@izmiran.troitsk.ru \\ ${ }^{2}$ Institute of Solar-Terrestrial Physics, Irkutsk, Russia, email: grechnev@iszf.irk.ru
}

\begin{abstract}
We summarize results of our recent studies of CME-associated EUV dimmings and coronal waves by 'derotated' fixed-difference SOHO/EIT heliograms at $195 \AA$ with 12 -min intervals and at $171,195,284,304 \AA$ with 6 -h intervals. Correctness of the derotated fixed-difference technique is confirmed by the consideration of the Bastille Day 2000 event. We also demonstrate that long narrow channeled dimmings and anisotropic coronal waves are typical of the complex global solar magnetosphere near the solar cycle maximum. Homology of large-scale dimmings and coronal waves takes place in a series of recurrent eruptive events. Along with dimmings coinciding entirely or partially in all four EIT bands, there exist dimmings that appear different, mainly in the transition-region line of $304 \AA$ and high-temperature coronal line of $284 \AA$.
\end{abstract}

Keywords. Sun: coronal mass ejections (CMEs), corona, UV radiation

\section{Introduction}

Strong reconfiguration of the magnetic fields during coronal mass ejections (CMEs) are known to be accompanied, in particular, by such large-scale phenomena as dimmings and coronal waves (e.g., Thompson et al., 1998; Zarro et al., 1999; Gopalswamy \& Thompson, 2000; Webb, 2000; Hudson \& Cliver, 2001). Dimmings, or transient coronal holes, are regions of temporary depressions of soft X-ray and EUV emissions formed after a CME near the eruptive center, for example, at the periphery of pre-eruptive sigmoid structures. Analysis of heliograms recorded with the EUV Imaging Telescope (EIT; Delaboudinière et al., 1995) aboard SOHO and with the Soft X-ray Telescope (SXT; Tsuneta et al., 1991) aboard Yohkoh showed that dimmings can exist during many hours and cover a large portion of the solar disk. Coronal waves are emitting fronts that are observed fairly often ahead the developing dimmings and propagate from the eruptive center with a speed of several hundreds $\mathrm{km} / \mathrm{s}$.

Usually, both the dimmings and coronal waves are studied with so-called runningdifference images obtained by consecutive subtracting of each current heliogram from the following one. Such running-difference images emphasize changes of the brightness, location, and configuration of observed structures between two subsequent heliograms, but inevitably result in some artifacts. In this paper, we present some results of our recent studies of dimmings and, to a lesser degree, coronal waves, obtained with SOHO/EIT data by means of the method of 'derotated' fixed-difference images (Chertok \& Grechnev, 2003a,b, 2005a,b). Those images are formed in two stages: first, the solar rotation is compensated using three-dimensional rotation of all images ('derotation') to the time of a pre-event base heliogram, and then this heliogram is subtracted from all following ones.

In the next section, the method of data processing is outlined. Then we demonstrate reasonableness of the derotated fixed-difference technique with an example of one of the famous eruptive events. Section 4 deals with anisotropic channeled dimmings characteristic of a complex topology of the global solar magnetosphere near the maximum of the 
solar activity. Homology of large-scale dimmings and anisotropy of coronal waves are described in Section 5. Section 6 is devoted to dimming manifestations in four SOHO/EIT different-temperature bands of $171,195,284$, and $304 \AA$. Some concluding remarks are given in Section 6.

\section{Data processing}

First, we process all SOHO/EIT images using the IDL $†$ routine EIT_PREP.PRO. This routine performs background subtraction, degridding images, and the flat-field correction. Next, the images are properly oriented, centered, and variations of the exposure time are corrected. We also resize all the images to $512 \times 512$ pixels, which is sufficient to study such large-scale features as dimmings, but reduces the time consumption in data processing.

In the $512 \times 512$ pixel EIT images with a pixel size of $5^{\prime \prime} .24$, the solar rotation effect appears to be almost inessential when difference images (as running ones) are obtained by subtracting heliograms taken $12 \mathrm{~min}$ apart. However, the solar rotation becomes important when the interval between frames exceeds tens of minutes, as in the case of the fixed-difference images. In particular, the displacement of any feature due to the solar rotation produces in the difference image a false eastern edge of the opposite contrast.

To avoid the appearance of false darkenings and brightenings, before the formation of the difference images, we compensate the rotation of the Sun by means of the 'derotation' of all heliograms to the same selected time, usually to the time of the reference frame before the event. In the software used, the solid rotation is applied to the whole visible solar hemisphere at the photospheric radius. Such a rotation is not perfect, because in the reality the solar rotation is not solid, and $3 \mathrm{D}$ structures observed in the EUV range comprise some altitude interval at larger heliocentric distances. However, possible errors due to the solid rotation of the sphere at $1 R_{\odot}$ are small and cannot change noticeably the observed picture of dimmings and other features, especially for events occurring in the central part of the solar disk.

To 'rotate' a solar image around the solar polar axis, we first convert the rectangular sky-plane coordinates of pixels on the visible solar surface into the longitude and latitude. Then we transform the longitudes according to the solar rotation during the required time interval, and convert the longitudes and latitudes to the sky-plane coordinates again. Finally, we transform the solar image to the last coordinates. The coordinate-conversion IDL routines were developed by M. J. Aschwanden.

When displaying the difference images, we limit their brightness with symmetric bipolar thresholds. In this way, show up negative dimmings, which are faint (typically 100-200 counts) with respect to very bright flare regions (some 1000 counts) observed simultaneously in the same frames.

To study dimmings quantitatively, we compute time profiles in a few small regions on the solar disk in the same way as Zarro et al. (1999) did. These light curves allow estimating the depth of the dimmings and show the evolution of their various parts as well as their temporal relation.

\section{Reasonableness of derotated fixed-difference images}

Correctness of the derotated fixed-difference images in comparison with the runningdifference images, especially for dimmings, can be illustrated by the famous Bastille Day 2000 event referring to the paper by Andrews (2001). The author of this paper considers,

$\dagger$ IDL is the trademark of the Research Systems, Inc. 

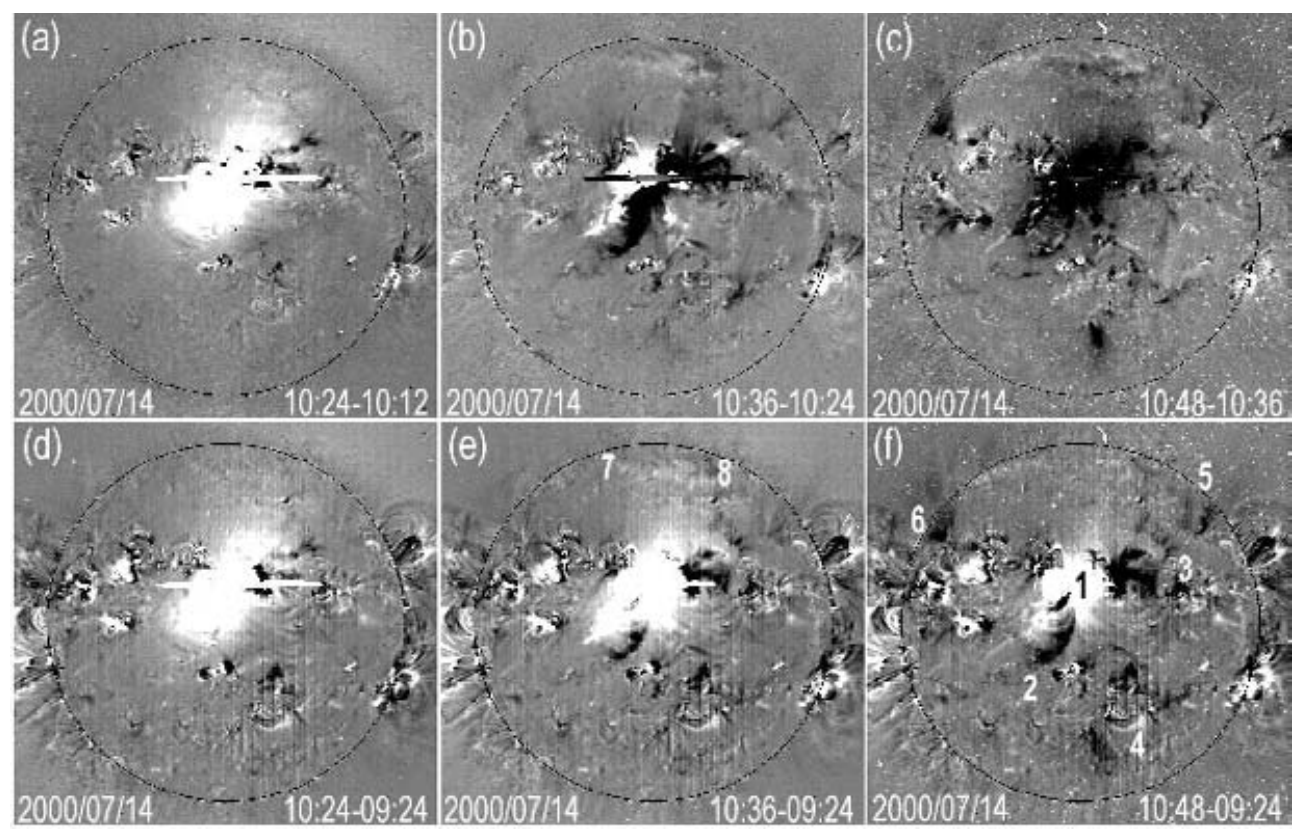

Figure 1. SOHO/EIT $195 \AA$ running-difference $(\mathrm{a}-\mathrm{c})$ and derotated fixed-difference $(\mathrm{d}-\mathrm{f})$ full-disk images of the Bastille Day 2000 event. The real picture of the dimmings is displayed by the fixed-different images, but not running-difference ones.

in fact, running-difference EIT images at $195 \AA$ similar to those presented in the upper row of Figure 1, but interprets them as fixed-difference images. This brings him to wrong conclusions. In particular, he correctly notes that a large dark region in frame (c) roughly corresponds to the area that was bright at 10:24 UT (frame (a)). However, his conclusion that this dark region is a real dimming, is erroneous. As a matter of fact, the greater central part of this darkening that appears in the running-difference image (c) is due to the intensity decrease of the flare brightening happened after 10:24 UT, and therefore represents a false dimming.

The real picture of dimmings displayed by the fixed-difference images shown in Figure 1 (d-f) is quite different (Chertok \& Grechnev, 2005a). Two main pronounced dimmings extend southward and westward of the bright structure above the eruptive center. The south transequatorial J-like dimming 1-2 is observed in conjunction with several emitting pre-event loops which increased their brightness as a result of the flare. The western composite dimming 1-3 covers an area between the eruptive center and remote active region. Besides, there are several additional dimming elements extending particularly to the southwestern region 4 as well as to the northwestern (5) and northeastern (6) limbs. The north emitting structure $7-8$ in frame (e) can be identified as a coronal wave front.

\section{Channeled dimmings}

There were halo CME events in which both coronal waves and dimmings developed more or less symmetrically in all directions from the eruption center as quasi-isotropic disturbances. It has been noted that such events occurred mainly in 1997-1998, i.e., at the ascending phase of the $23^{\text {rd }}$ cycle when the global solar magnetosphere was comparatively simple. For example, one of well-known events of that kind, May 12, 1997, was observed when a single active region was present at the disk (Thompson et al., 1998). On the 


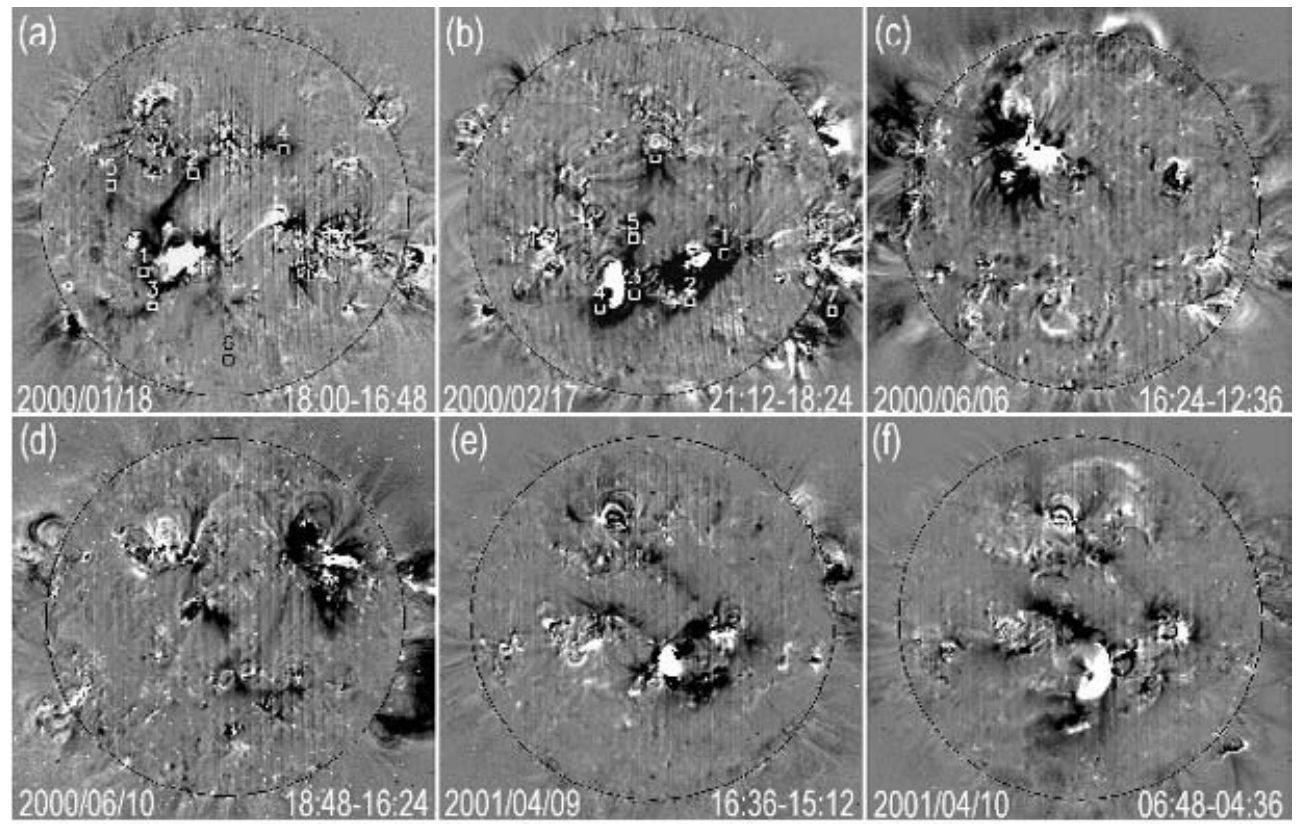

Figure 2. Examples of CME-associated events with developed channeled dimmings as visible at $195 \AA \mathrm{SOHO} / \mathrm{EIT}$ derotated fixed-difference images.
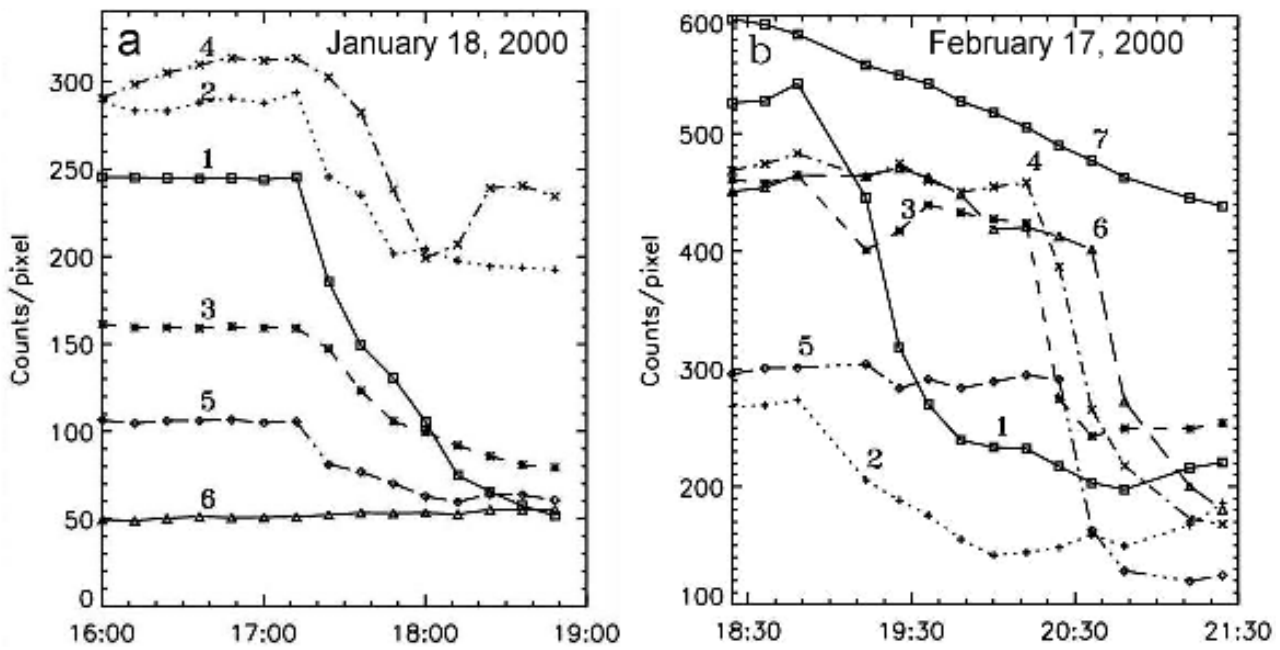

Figure 3. Brightness variations at $195 \AA$ during events of January 18, 2000 and February 17, 2000 for several $53^{\prime \prime} \times 53^{\prime \prime}$ areas labeled correspondingly in Figure $2(\mathrm{a}, \mathrm{b})$.

contrary, our analysis of halo CME events for 2000-2002 (Chertok \& Grechnev, 2003a) revealed that strongly anisotropic dimmings stretching along narrow extended features (channels) are typical of the complex solar magnetosphere at the cycle maximum epoch (cf. Delannée, 2000).

Several examples of such channeled dimmings are given in Figure 2 where derotated fixed-difference images with multi-hour intervals between the reference and current heliograms are shown. One can see that in each event, the channeled dimmings have a global character, with a length exceeding the solar radius; they are transequatorial and extend 

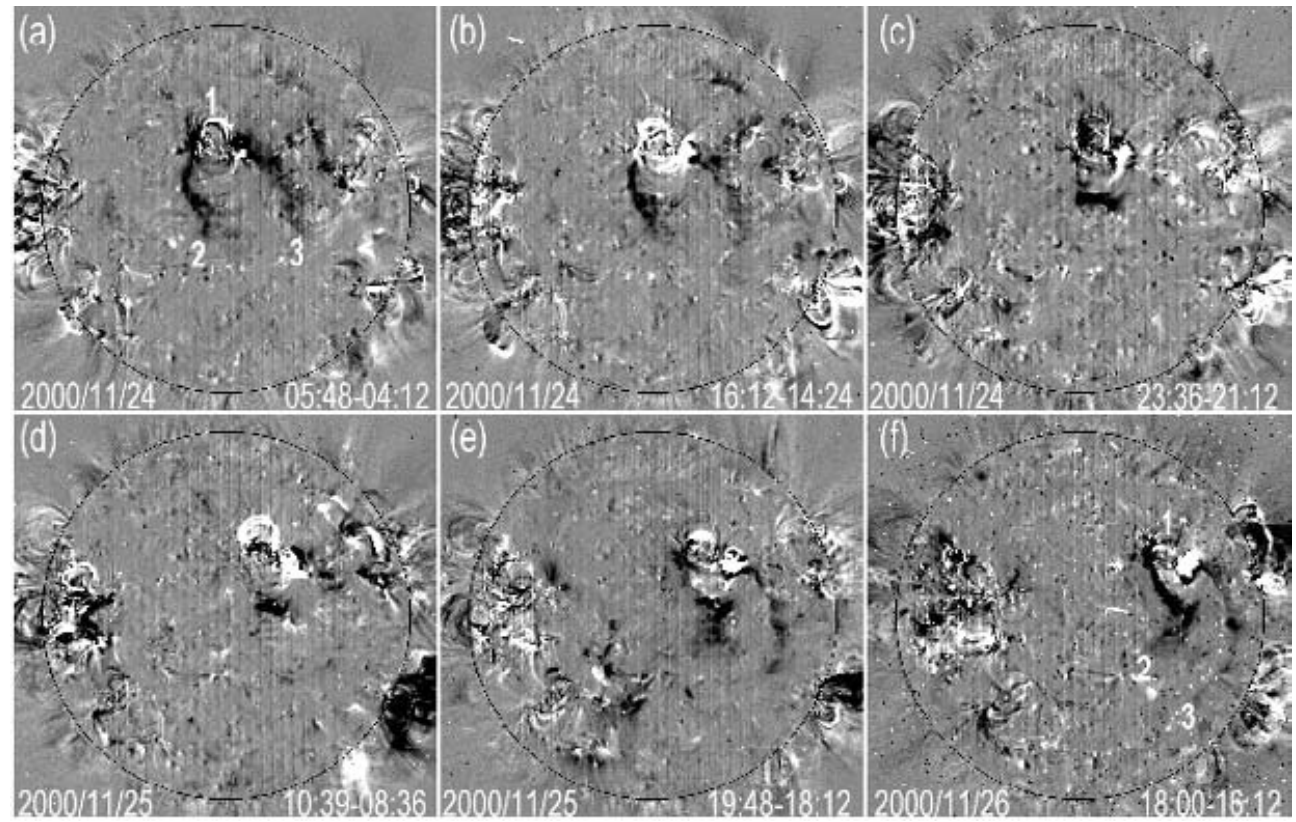

Figure 4. SOHO/EIT fixed-difference images at $195 \AA$ illustrating homology of large-scale dimmings in events of the November 2000 series.

between remote active regions. By their characteristics, such as the intensity depletion and lifetime, the channeled dimmings do not differ significantly from compact dimmings adjoining to eruptive centers. This is illustrated at Figure 3 where the depth and temporal changes in some selected $53^{\prime \prime} \times 53^{\prime \prime}$ areas are presented for two events shown in Figure $1(\mathrm{a}, \mathrm{b})$. The corresponding areas and time profiles are labeled by the same digits. It follows from these data that the depth of the channeled dimmings reaches several tens per cent, they develop for several tens of minutes, and their lifetimes are, at least, several hours. In channeled-dimming events, coronal waves are also anisotropic, and they propagate within a restricted sector of the disk (see next Section).

\section{Homology of dimmings and coronal waves}

Two events shown in Figure 2 (e,f) occurred from the same eruptive center with a time interval of about $14 \mathrm{~h}$. They show rather obvious similarity in the location and topology of the large-scale channeled dimmings. Such homology of CME-associated disturbances appears to be a general property of seriate eruptive events.

Chertok et al. (2004a) studied large-scale activity on the solar disk associated with a November 24-26, 2000 series of five X-class and one M3.5-class recurrent flares and halo CMEs. The analysis showed that those eruptive events were homologous not only by their flare and CME characteristics, as Nitta and Hudson (2001) described, but also in terms of their large-scale CME-associated manifestations. SOHO/EIT $195 \AA$ derotated fixed-difference images (Figure 4) show that two channeled transequatorial dimmings 1-2 and 1-3, extending southward of the eruptive center, predominated in practically all events of this series. These two dimmings appear to outline footpoints of a coronal arcade observed above an $\mathrm{H} \alpha$ filament, with the eastern dimming 1-2 stretching along the western boundary of a narrow preexisting coronal hole $(\mathrm{CH})$. 

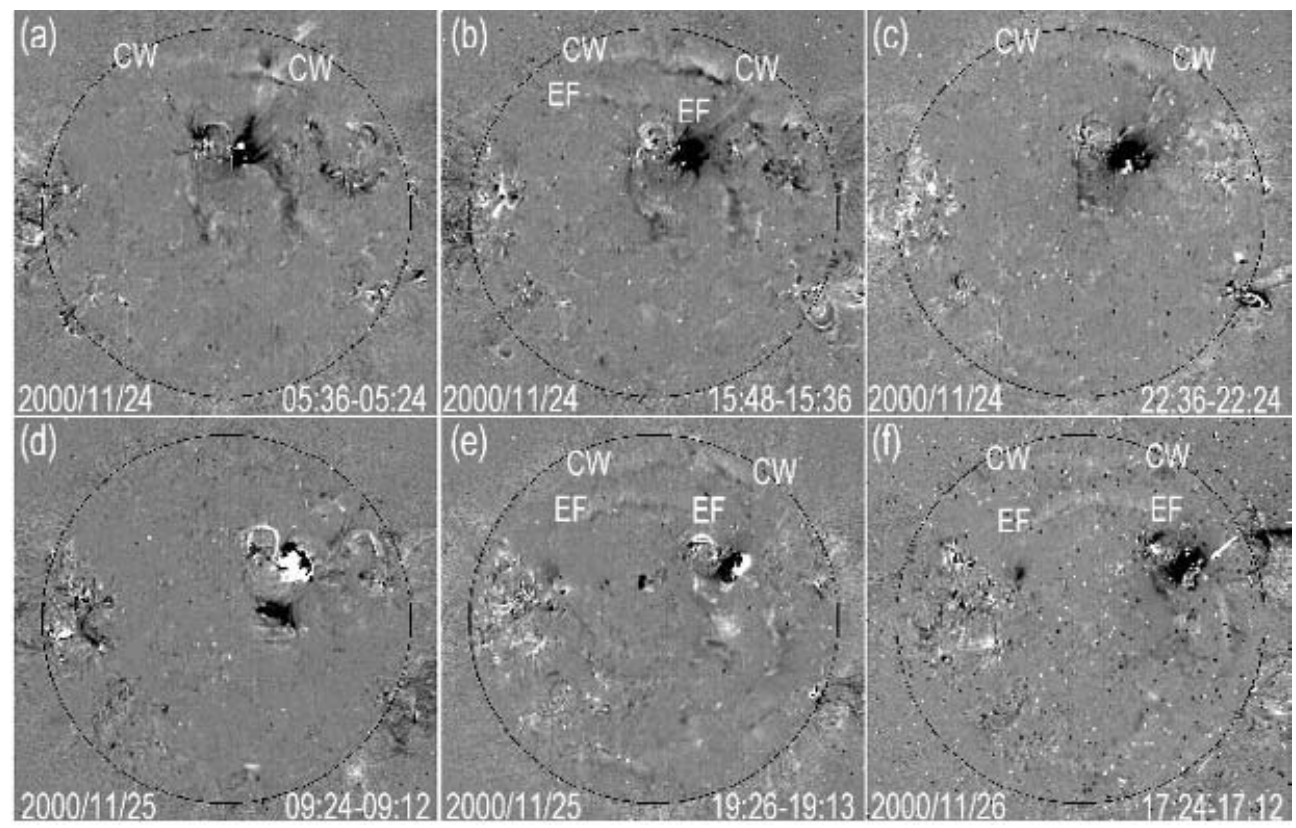

Figure 5. SOHO/EIT running-difference images with 12 -min intervals at $195 \AA$ showing homologous coronal waves $(\mathrm{CW})$ and quasi-stationary emitting fronts $(\mathrm{EF})$ in events of the November 2000 series.

Large-scale homology of the events of this series was manifest in accompanying coronal waves. Because they are relatively weak, we use for the illustration running-difference images keeping in mind the above remarks. As one can see from Figure 5 (and especially with corresponding movies), all of the events (except perhaps event d) were accompanied by similar coronal waves $(\mathrm{CW})$. In these running-difference images, coronal waves are visible as bright fronts, and dark fronts behind them mark their locations in preceding images. The main property of these disturbances is that in the different events they propagated anisotropically inside the same restricted angular sector. It is essential that, whereas the main dimmings described above developed southward of the eruptive center 1, all observed coronal waves propagated mainly in the northern direction. Moreover, the second and also similar emitting fronts (EF) were observed, at least, in events b, e, $\mathrm{d}$, and f. A peculiarity of these emitting fronts also seen with movies is that they almost do not propagate and, instead, occupy more or less stationary positions on the disk (cf. Delannée, 2000).

Note that remarkable large-scale homology, especially for dimming patterns, also took place in a series of extremely powerful flares and large CMEs associated with a complex of three active regions in October-November 2003 (Chertok \& Grechnev, 2005b).

\section{Dimmings in four different-temperature bands}

In the standard mode of SOHO/EIT observations, full-disk heliograms are produced almost every $12 \mathrm{~min}$ at $195 \AA$, but only 4 times per day near $01,07,13$, and 19 UT at 171 , 284 , and $304 \AA$. Let us remind that three coronal channels of 171,195 , and $284 \AA$ with predominating lines FeIX/X, FeXII, and FeXV are sensitive to plasma temperatures of 1.2, 1.5 , and $2.0 \mathrm{MK}$, respectively, while the $304 \AA$ filter passes the transition-region line HeII (0.02-0.08 MK) and much weaker coronal component SiXI (1.6 MK) (Delaboudinière 


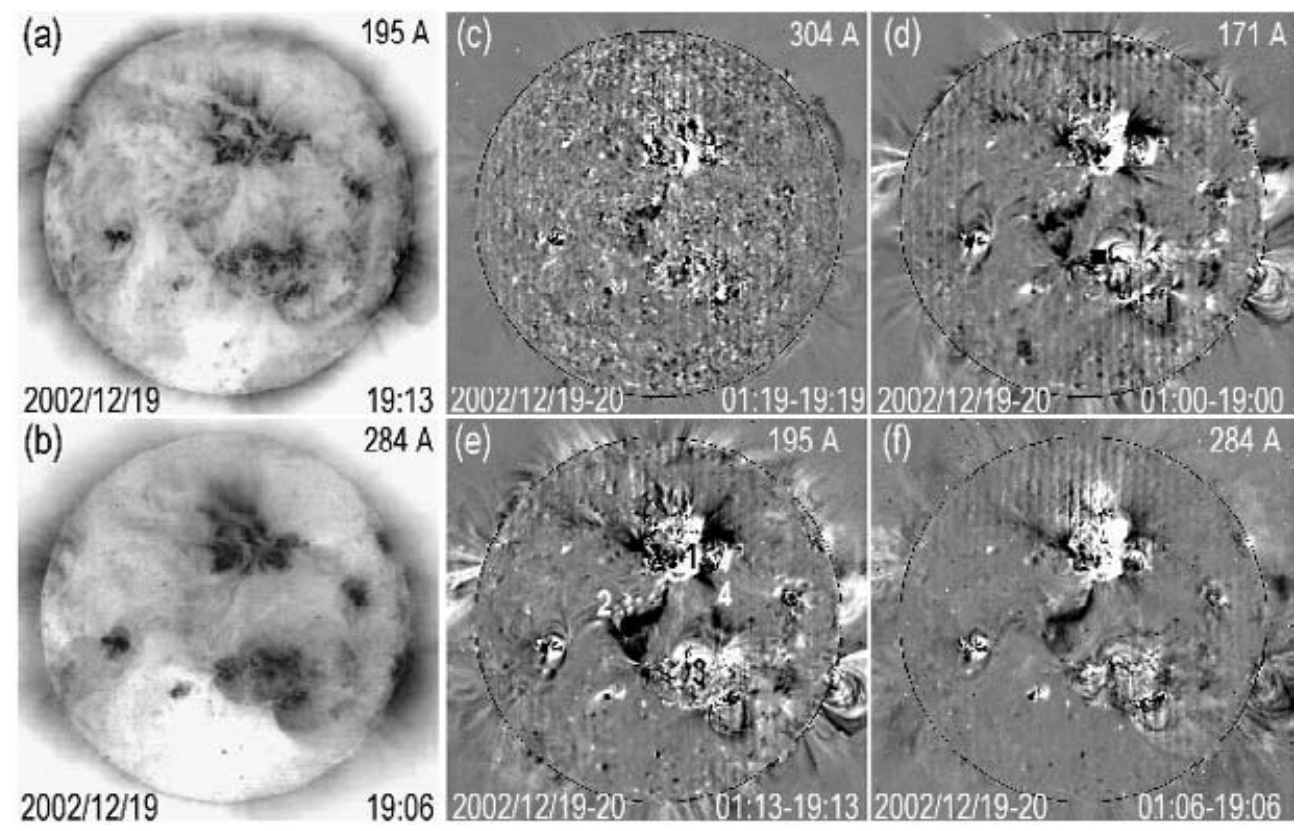

Figure 6. SOHO/EIT images related to the eruptive event of December 19, 2002: (a,b) Negative pre-event heliograms at 195 and $284 \AA$; (c-f) Derotated difference images with a 6-hour interval illustrating dimmings in four EIT bands.

et al., 1995). Due to the derotation procedure, we are able to form difference images with 6 -h or 12-h intervals in all four bands simultaneously, and therefore to study dimming manifestations in different-temperature lines (Chertok \& Grechnev, 2003b).

The eruptive event of December 19, 2002 (Figure 6) represents an example of CMEinitiated global dimmings, some of which are almost identical in all three coronal lines, with a pronounced dimming manifestation also in the transition-region line. The eastern transequatorial dimming 1-2-3 extending from the northern eruptive center to the remote southern active region coincides at $171,195,284 \AA$ and is clearly visible at $304 \AA$. At the same time, the western fragmentary transequatorial dimming is very similar in two moderate-temperature coronal lines 171 and $195 \AA$, but is practically absent in hightemperature coronal line $284 \AA$ as well as in the transition-region line $304 \AA$.

In June 10, $2000 \mathrm{CME}$ event (Figure 7), again, very similar large-scale channeled dimmings extending through the disk are observed in both the moderate-temperature coronal lines 171 and $195 \AA$. One transequatorial dimming structure 1-2 stretches from northeastern outskirts of the eruptive center in the southwestern direction. The second similar structure is formed by dimmings located along the transequatorial line 3-45 interconnecting remote active regions. The most remarkable feature of this event is that both the transequatorial dimming structures visible at 171 and 195 Apractically are not manifest in the high-temperature coronal line $284 \AA$. The dimming manifestations are comparatively poor also in the transition-region line $304 \AA$. Only some small-area analogs of the coronal dimmings, located near the eruptive center 1 and partly near other involved active regions, are distinguished on the disk in this low-temperature line. A sole exception is the northern high-latitude dimming ribbon $6-7$ also visible at two moderate-temperature coronal lines of 171 and $195 \AA$.

During the April 29, 1998 event (Figure 8), the eruptive center 1 was, in fact, the only active region on the disk, but it was adjacent to a large $\mathrm{CH}$ that occupied nearly the 

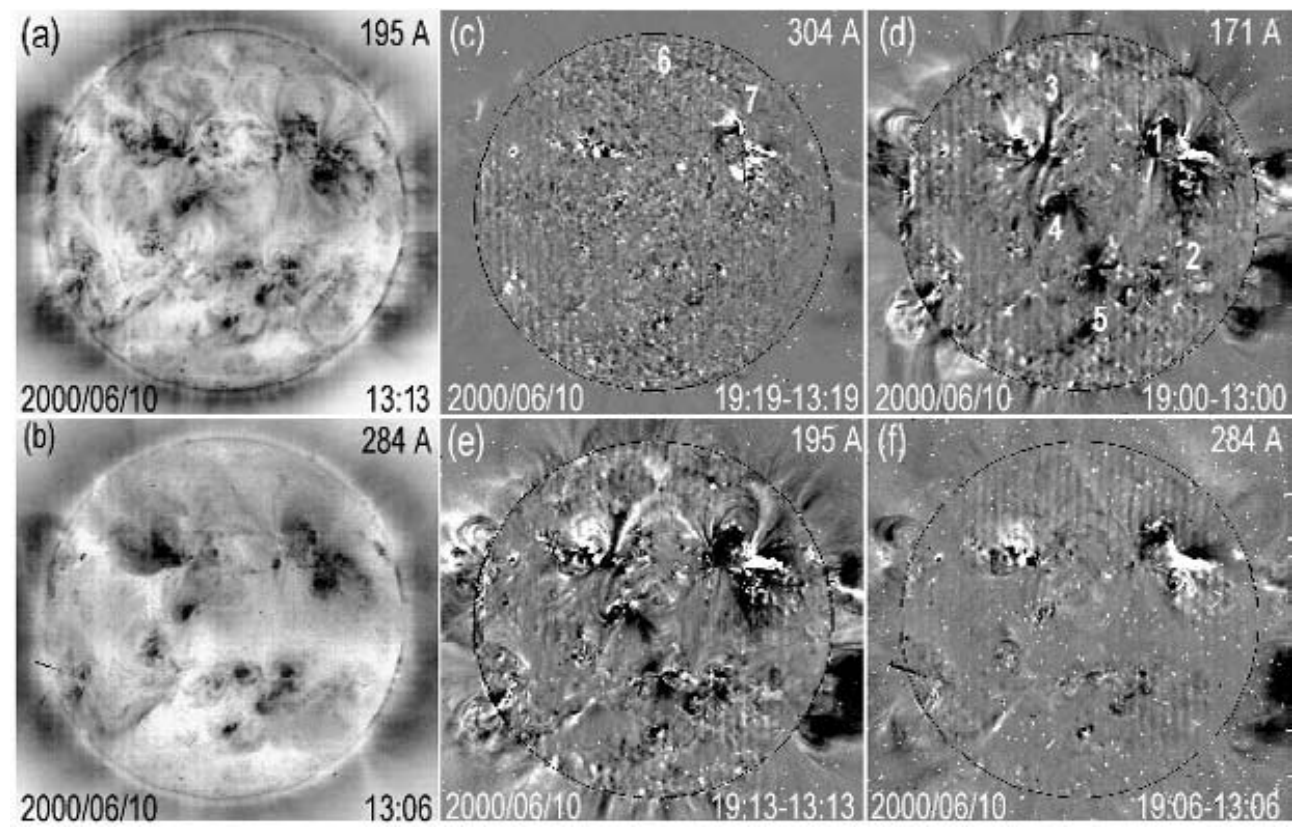

Figure 7. Same as in Figure 6 for the CME event of June 10, 2000.

whole central zone of the disk. At $195 \AA$, the main dimmings developed in the direction of the northeastern limb: the deepest transequatorial dimming 1-2 formed in the immediate vicinity of $\mathrm{CH}$ extends toward northern region and joins with the eastern meridional dimming 3-4. As usually, similar overall dimming structure is also observed at $171 \AA$. One of the minor differences from the $195 \AA$ pattern is that dimming $1-2$ is somewhat broadened in the longitudinal direction in the immediate vicinity of the eruption center 1 , and that only individual fragments are visible at the place of the eastern meridional dimming 3-4. The main transequatorial dimming 1-2 is also present in the high-temperature $284 \AA$ coronal line. A remarkable peculiarity of this event is that a large dark area was observed in $304 \AA$ band within the pre-event $\mathrm{CH}$ without any counterparts in coronal lines. This pronounced dimming $1-5-6$ starts from the eastern side of the eruptive center, covers an extensive portion of $\mathrm{CH}$ stretching westward to point 5 , then bends and extends southeastward to point 6 located near the southern boundary of $\mathrm{CH}$. It is reasonable to suppose that the two-component structure of the CME observed in this case was connected with the described dimming pattern: perhaps the bright northeastern CME component corresponds to the coronal dimming, while the dimming-like feature visible in $304 \AA$ band corresponds to the southwestern CME component.

\section{Concluding remarks}

Thus, due to the derotation technique, we are able to form fixed-difference EUV images with diverse time intervals between the base and current heliograms and in differenttemperature lines. This allows us obtaining real pictures of long-living dimmings and detecting some interesting features of CME-associated large-scale disturbances.

The analysis of derotated fixed-difference images has revealed, in particular, that when the global solar magnetosphere is complex, which is typical of the solar cycle maximum, dimmings are often anisotropic and spread along narrow extended channel-like structures. In halo CME events, these channeled dimmings often have a global character, and 


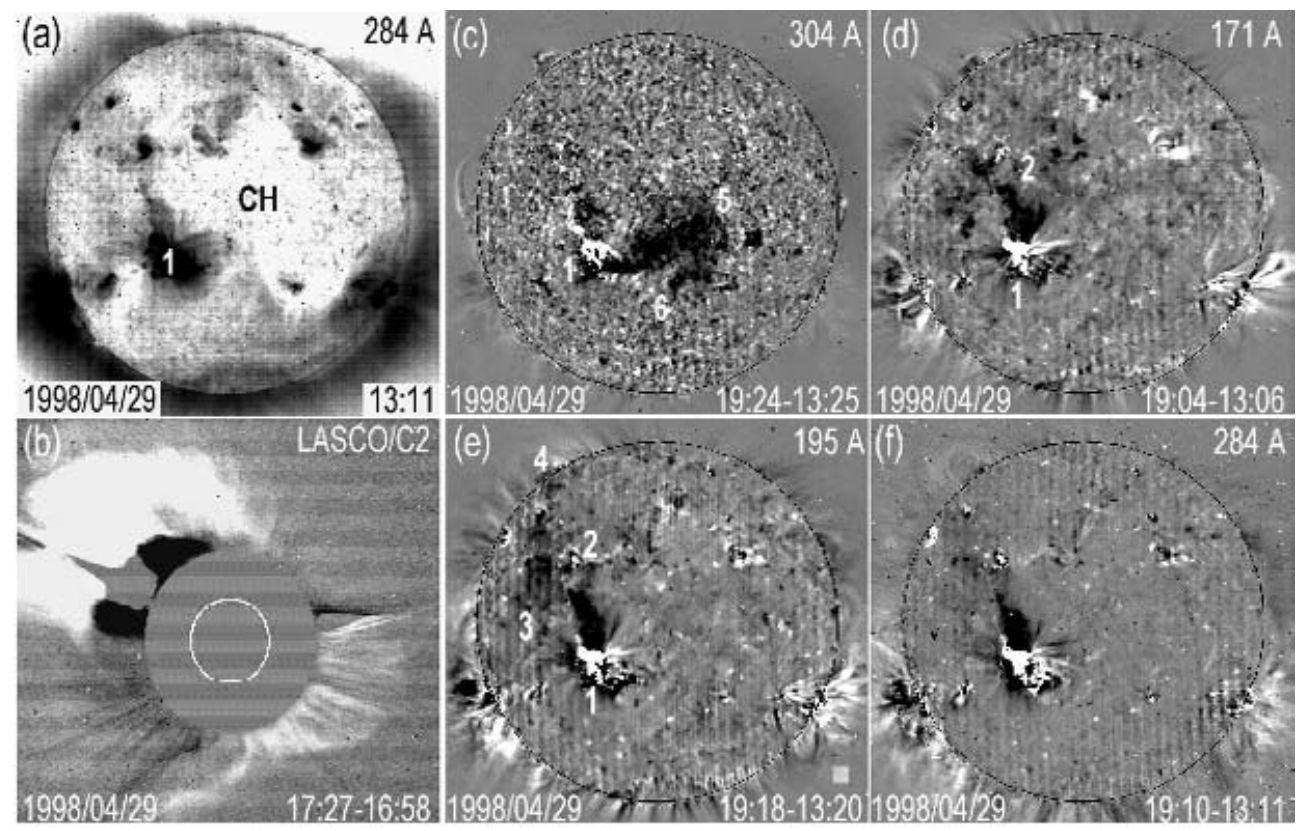

Figure 8. SOHO/EIT \& LASCO images related to the eruptive event of April 29, 1998: (a) Negative pre-event heliogram at $284 \AA$ showing a large central $\mathrm{CH}$; (b) Two-component CME; (c-f) Derotated difference images with a 6-hour interval illustrating dimmings in four EIT bands.

can embrace almost entire visible solar disk extending between remote active regions located on different sides from the helioequator. The EUV intensity depletions in the channeled dimmings are comparable to or somewhat less than those in the isotropic and patchy dimmings. Herewith, coronal waves are also observed as anisotropic disturbances propagating within a restricted angular sector.

Large-scale homology of dimmings and coronal waves in repetitive events originating recurrently from the same eruptive center has been found. Dimming homology means that the same large-scale structures, exposed to a strong CME-caused restructuring, can restore their plasma characteristics and emissivity at a time scale of 10-15 h. As for observed coronal wave homology and anisotropy, they seem be determined by the structure of the global solar magnetosphere and to confirm that the corresponding disturbances tend to avoid strong magnetic fields, particularly active regions (e.g., Ofman and Thompson, 2002).

The analysis of dimmings in four SOHO/EIT channels based on derotated fixeddifference images taken at intervals of $6 \mathrm{~h}$ and sometimes of $12 \mathrm{~h}$ indicates that dimmings are normally strongly pronounced and have similar large-scale structures in the moderate-temperature 171 and $195 \AA$ coronal lines. The dimmings can be also visible in the higher-temperature $284 \AA$ line, although there are cases when some coronal dimmings, particularly similar to transequatorial interconnecting loop structures (see Khan and Hudson, 2000), are not pronounced in this line. In addition, some coronal dimmings are visible in the transition-region line $304 \AA$, especially those adjacent to the eruptive center. Sometimes $304 \AA$ dimming-like features are observed without coronal counterparts.

The coinciding dimmings in three coronal lines or sometimes in all four spectral bands support their interpretation as a result of full or partial opening of magnetic field lines 
in some areas and structures in the course of CME processes accompanied by evacuation of plasmas from those structures (e.g., Thompson et al., 1998; Zarro et al., 1999; Harrison et al., 2003; Harra \& Sterling, 2001). At the same time, different appearance of dimmings, mainly, in the transition-region line $304 \AA$ and in the highest-temperature coronal line $284 \AA$, suggests that temperature variations can also play an important role in the formation of some dimming structures.

The fact that dimmings, both compact and extended, are also observed sometimes at $304 \AA$ shows that the transition region plasma is also involved in CME processes. Transition-region dimmings result from the opening of the magnetic fields and plasma outflow from originally closed, low-lying structures, i.e. a transient 'hole' is formed down to the transition region. One cannot also rule out occultation effects in absorbing material of erupting filaments for short-term and moving dimmings (Slemzin et al., 2005). Such an effect is likely responsible for the $304 \AA$ dimming observed without coronal counterparts.

On the other hand, long evolution times of many dimmings along with gradual fade of emitting structures sometimes observed suggest the possibility of other mechanisms, e.g., CME-caused suppression of either heating of coronal structures, or mass supply into them (Uralov \& Grechnev, 2004).

The outlined technique of derotated fixed-difference images has also been applied to the analysis of EUV data from the SPIRIT telescopes aboard the CORONAS-F spacecraft (Chertok et al., 2004b; Slemzin et al., 2004).

Various difference images and corresponding movies for many CME events, including ones described in this paper, are presented at the Web site

http://helios.izmiran.troitsk.ru/lars/Chertok/.

\section{Acknowledgements}

We are grateful to SOHO/EIT team members for data used in this research. SOHO is a project of international cooperation between ESA and NASA. This work is supported by the Russian Foundation of Basic Research (grants 03-02-16049, 03-02-16591) and the Ministry of Education and Science (grants 447.2003.2, 1445.2003.2).

\section{References}

Andrews, M. D. 2001, Solar Phys., 204, 181

Chertok, I. M. \& Grechnev, V. V. 2003a, Astron. Reports 47, 139

Chertok, I. M. \& Grechnev, V. V. 2003b, Astron. Reports 47, 934

Chertok, I. M. \& Grechnev, V. V. 2005a, Solar Phys., accepted

Chertok, I. M. \& Grechnev, V. V. 2005b, Astron. Reports 49, 155

Chertok, I. M., Grechnev, V. V., Hudson, H. S., \& Nitta, N. V. 2004a, J. Geophys. Res. 109, doi:10.1029/2003JA010182

Chertok, I. M., Slemzin, V. A., Kuzin, S. V. et al. 2004b, Astron. Reports 48, 407

Delaboudinière, J.-P., Artzner, G. E., Brunaud, J. et al. 1995, Solar Phys. 162, 291

Delannée, C. 2000, Astrophys. J. 545, 512

Gopalswamy, N. \& Thompson, B. J. 2000, J. Atmos. Sol-Terr. Phys. 62, 1457

Harrison, R. A., Bryans, P., Simnett, G. M. et al. 2003 Astron \& Astrophys. 400, 1071

Harra, L. K., Sterling, A. C. et al. 2001 Astrophys. J. Lett. 561, 216

Hudson, H. S. \& Cliver, E. W. 2001 J. Geophys. Res. 106, 25199

Khan, J. I. \& Hudson, H.S. 2000, Geophys. Res. Lett. 27, 1083

Nitta, N. V. \& Hudson, H. S. 2001, Geophys. Res. Lett. 28, 3801

Ofman, L. \& Thompson, B. J. 2002, Astrophys. J. 574, 440

Slemzin, V., Zhitnik, I. A., Ignat'ev, A.P. et al. 2005, this volume, 119

Thompson, B. J., Plunkett, S. P., Gurman, J. B. et al. 1998 Geophys. Res. Lett. 25, 2465-2468. 
Tsuneta, S., Acton, L., Bruner, M. 1991, Solar Phys. 136, 37

Uralov, A. M. \& Grechnev, V. V., Hudson, H. S. 2005, J. Geophys. Res., accepted

Webb, D. F. 2000, J. Atmos. Sol-Terr. Phys. 62, 1415

Zarro, D. M., Sterling, A. C., Thompson, B.J. et al. 1999 Astrophys. J. 520, L139

\section{Discussion}

GopaLSWAMY: You mentioned several possible mechanisms of dimming. Especially, there were two possibilities (wave process and plasma out flow) now debated. In my opinion, a CME process contains both. The plasma outflow is the CME and the wave part surrounds the CME.

Grechnev: I listed various possible mechanisms to explain different darkening phenomena. It is possible that the magnetic configuration opens, and then some part is taken away by CME, and some by waves. However, 1) long-term deepening of dimmings with impulsive launch of CMEs and 2) observations of fading closed structures suggest also suppression of either heating, or mass supply into closed coronal structures or both. So different mechanisms can contribute to dimming phenomena.

NinDOS: How did you correlate the EIT channeled dimmings with the large-scale patchy channeled features sometimes observed at $17 \mathrm{GHz}$ with Nobeyama Radioheliograph?

GrEChNEv: The bright chains in the microwave emission were really found by the first author of this talk, I. Chertok, before the finding channeled dimmings. However, we have not yet studied associations of channeled dimmings with other extended structure, but we want to do it. The only thing I can state now is that dimmings appear along features between active regions, sometimes along transequatorial loops, and often embrace almost all the visible solar disk.

SCHMIEDER: 1. Comments: The anisotropy of the EIT waves during the maximum solar cycle could be explained by the presence of active regions and inversion magnetic field lines.

2. The dimming observed in $304 \AA$ can be due to the absorption of the EUV emissions. At this wavelength the optical thickness of cool material is larger than the optical thickness in $\mathrm{H} \alpha$. This implies that cool material is not visible in $\mathrm{H} \alpha$ but could be efficient for the absorption mechanism by He Lyman continuum.

Grechnev: 1. Yes, it is definitely connected with the structure of the global solar magnetosphere, as a said.

2. I just put it No.1 in my Concluding Remarks: occultation by absorbing material. This is definitely caused by some absorption, but it is a question what kind of absorption works: non-resonant or resonant. Of course it is an interesting question to study.

ZHukov: 1. The first comment is about your statement that dimmings could be due to Doppler effect. EIT is not a spectrometer, its bandpasses are wide and contain a lot of spectral lines, so I don't think this explanation is plausible.

2. I think that the difference of dimming patterns in coronal and transition region bandpasses is caused by the fact that you use low-cadence (6 hours) observations in $304 \AA ̊$ bandpass.

GRECHNEV: 1. I only mentioned the possible interpretation that was proposed. 
2. No, there is another reason, I think. The dimming in the $304 \AA$ band is likely due to absorption in remnants of an ejected filament, but this absorption is not so efficient in coronal emission lines (171 and $195 \AA$ ).

Kouтснмy: When de-rotating your images from a time sequence, did you assume a rigid rotation and what about using some "differential" rotation?

Grechnev: Yes, we apply solid rotation. The differential rotation is hardly important for intervals of several hours. Another related question is that we rotate the sphere of a fixed radius, but the coronal structures have some extent in altitude. However, according to our estimation, both these circumstances do not significantly affect such relatively long-scale features, as dimmings.

Delaboudiniere: Dimmings in $304 \AA$ are due to occultation by filament ejected material and are totally different from coronal dimmings (not at the same location!!).

Grechnev: Yes, this is just the first point from my slide "Nature of dimmings": Occultation by absorbing material. I list various possible reasons for various darkening effects.

JingxiU WANG: After the careful data deduction, your results seem to not support the wave interpretation for dimming? Is this true?

GreChNEv: No, waves definitely are present in several eruptive events. We were against mixing methodical artifacts with waves and dimmings which really exist, and their real structure with that one which appears in running-difference images, which are actually the temporal derivative of the structure observed. 